\title{
Systolic pressure
}

National Diabetes Information Clearinghouse (NDIC)

\section{Definitions}

Artery

Defined by National Diabetes Information Clearinghouse (NDIC)

Blood pressure

Defined by National Diabetes Information Clearinghouse (NDIC)

Source

National Diabetes Information Clearinghouse (U.S.). (2009). The diabetes dictionary. [Bethesda, Md.]: U.S. Dept. of Health and Human Services, National Institutes of Health, National Institute of Diabetes and Digestive and Kidney Diseases, National Diabetes Information Clearinghouse.

The blood pressure when the heart pushes blood out into the arteries. 